\title{
Closed Analytic Formulas for the Approximation of the Legendre Complete Elliptic Integrals of the First and Second Kinds
}

\author{
Richard Selescu \\ "Elie Carafoli" National Institute for Aerospace Research - INCAS \\ (under the Aegis of the Romanian Academy), \\ Department of Aerodynamics, Blvd. Iuliu Maniu, No. 220, Code 061126, \\ Sector 6, Bucharest, Romania
}

\begin{abstract}
The author proposes two sets of closed analytic functions for the approximate calculus of the complete elliptic integrals of the first and second kinds in the normal form due to Legendre, the respective expressions having a remarkable simplicity and accuracy. The special usefulness of the proposed formulas consists in that they allow performing the analytic study of variation of the functions in which they appear, by using the derivatives. Comparative tables including the approximate values obtained by applying the two sets of formulas and the exact values, reproduced from special functions tables are given (all versus the respective elliptic integrals modulus, $k=\sin \theta$ ). It is to be noticed that both sets of approximate formulas are given neither by spline nor by regression functions, but by asymptotic expansions, the identity with the exact functions being accomplished for the left end $k$ $=0\left(\theta=0^{\circ}\right)$ of the domain. As one can see, the second set of functions, although something more intricate, gives more accurate values than the first one and extends itself more closely to the right end $k=1(\theta=$ $90^{\circ}$ ) of the domain. For reasons of accuracy, it is recommended to use the first set until $\theta=70^{\circ} .5 \mathrm{only}$, and if it is necessary a better accuracy or a greater upper limit of the validity domain, to use the second set, but on no account beyond $\theta=88^{\circ} .2$.
\end{abstract}

\section{INTRODUCTION - DEFINITIONS}

There are many interesting domains of the pure and applied mathematics in which appear one or both complete elliptic integrals of the first and second kinds in the normal form due to Legendre. So, in the dynamics of a constrained heavy particle, the period of oscillations in a vacuum of the simple pendulum is given by a complete elliptic integral of the first kind. In the geometry of plane curves, the length of an ellipse is given by a complete elliptic integral of the second kind. In the supersonic aerodynamics, the lift coefficient of a thin delta wing having subsonic leading edges is also given by a complete elliptic integral of the second kind. The following well-known relations define all these integrals. For the first kind complete integral we have

$\mathrm{K}(k)=\int_{0}^{\pi / 2} \frac{d \varphi}{\sqrt{1-k^{2} \sin ^{2} \varphi}}=\int_{0}^{1} \frac{d t}{\sqrt{\left(1-t^{2}\right)\left(1-k^{2} t^{2}\right)}}$,

and for the second kind one

$$
\mathrm{E}(k)=\int_{0}^{\pi / 2} \sqrt{1-k^{2} \sin ^{2} \varphi} d \varphi=\int_{0}^{1} \sqrt{\frac{1-k^{2} t^{2}}{1-t^{2}}} d t,
$$

where $k=\sin \theta$ is called modulus. They are calculated by expanding the integrands into series, integrating term-byterm and presented versus the modulus $k$ or frequently versus the angle $\theta$ in some mathematical tables [ $1-6]$. The values given in these tables allow performing the calculus for a given case (point), but not the analytic study of variation of the functions in which these integrals appear, by using the derivatives. In the following chapter are proposed two sets of closed analytic functions for the approximate calculus of both complete elliptic integrals. The first set is affected by the subscript 0 , while the second one by the subscript 1 .

\section{THE TWO SETS OF PROPOSED CLOSED} ANALYTIC FORMULAS

Using the complementary modulus too, $k^{\prime}=\sqrt{1-k^{2}}=\cos \theta$,

$$
\begin{aligned}
& \mathrm{K}_{0}(k)=\frac{\pi}{\sqrt[4]{1-k^{2}}}\left(1-\frac{1}{2 \sqrt{2}} \sqrt{\frac{1+\sqrt{1-k^{2}}}{\sqrt[4]{1-k^{2}}}}\right)=\pi\left(\frac{1}{\sqrt{k^{\prime}}}-\frac{1}{2 \sqrt{2}} \frac{\sqrt{1+k^{\prime}}}{k^{3 / 4}}\right), \text { or } \\
& \mathrm{K}_{0}(\theta)=\frac{\pi}{\sqrt{\cos \theta}}\left(1-\frac{1}{2} \frac{\cos \frac{\theta}{2}}{\sqrt[4]{\cos \theta}}\right)=\pi\left(\frac{1}{\sqrt{\cos \theta}}-\frac{1}{2} \frac{\cos \frac{\theta}{2}}{\cos ^{3 / 4} \theta}\right) \\
& \mathrm{E}_{0}(k)=\frac{\pi}{4} \sqrt[4]{1-k^{2}}\left(\frac{3}{2} \frac{1+\sqrt{1-k^{2}}}{\sqrt[4]{1-k^{2}}}-1\right)=\frac{\pi}{4}\left[\frac{3}{2}\left(1+k^{\prime}\right)-\sqrt{k^{\prime}}\right], \text { or }
\end{aligned}
$$$$
\mathrm{E}_{0}(\theta)=\frac{\pi}{4} \sqrt{\cos \theta}\left(3 \frac{\cos ^{2} \frac{\theta}{2}}{\sqrt{\cos \theta}}-1\right)=\frac{\pi}{4}\left(3 \cos ^{2} \frac{\theta}{2}-\sqrt{\cos \theta}\right) \text {; }
$$$$
\mathrm{K}_{1}(k)=\frac{\pi \sqrt{2}}{\sqrt{\left(1+k^{\prime}\right) \sqrt{k^{\prime}}}}\left(1-\frac{\sqrt[4]{2}}{4} \frac{1+\sqrt{k^{\prime}}}{\sqrt[4]{\left(1+k^{\prime}\right) \sqrt{k^{\prime}}}}\right) \text {, or }
$$$$
\mathrm{K}_{1}(\theta)=\frac{\pi}{\cos \frac{\theta}{2} \sqrt[4]{\cos \theta}}\left(1-\frac{1}{4} \frac{1+\sqrt{\cos \theta}}{\sqrt{\cos \frac{\theta}{2} \sqrt[4]{\cos \theta}}}\right) \text {; }
$$$$
\mathrm{E}_{1}(k)=\frac{\pi}{4}\left[\frac{3}{2}\left(1+\sqrt{k^{\prime}}\right)^{2}-\sqrt{2} \sqrt{1+k^{\prime}} \sqrt[4]{k^{\prime}}\right]-k^{\prime} \cdot \mathrm{K}_{1}(k) \text {, or }
$$

$\mathrm{E}_{1}(\theta)=\frac{\pi}{4}\left[\frac{3}{2}(1+\sqrt{\cos \theta})^{2}-2 \cos \frac{\theta}{2} \sqrt[4]{\cos \theta}\right]-\cos \theta \cdot \mathrm{K}_{1}(\theta)$. 
Table 1. Values of the functions $K$ (part one)

\begin{tabular}{|c|c|c|c|c|}
\hline$\theta\left({ }^{\circ}\right)$ & $k=\sin \theta$ & $\mathrm{K}(k)$ & $\mathrm{K}_{0}(k)$ & $\mathrm{K}_{1}(k)$ \\
\hline 0 & 0.00000 & 1.5708 & 1.5708 & 1.5708 \\
\hline 1 & 0.01745 & 1.5709 & 1.5709 & 1.5709 \\
\hline 2 & 0.03490 & 1.5713 & 1.5713 & 1.5713 \\
\hline 3 & 0.05234 & 1.5719 & 1.5719 & 1.5719 \\
\hline 4 & 0.06976 & 1.5727 & 1.5727 & 1.5727 \\
\hline 5 & 0.08716 & 1.5738 & 1.5738 & 1.5738 \\
\hline 6 & 0.10453 & 1.5751 & 1.5751 & 1.5751 \\
\hline 7 & 0.12187 & 1.5767 & 1.5767 & 1.5767 \\
\hline 8 & 0.13917 & 1.5785 & 1.5785 & 1.5785 \\
\hline 9 & 0.15643 & 1.5805 & 1.5805 & 1.5805 \\
\hline 10 & 0.17365 & 1.5828 & 1.5828 & 1.5828 \\
\hline 11 & 0.19081 & 1.5854 & 1.5854 & 1.5854 \\
\hline 12 & 0.20791 & 1.5882 & 1.5882 & 1.5882 \\
\hline 13 & 0.22495 & 1.5913 & 1.5913 & 1.5913 \\
\hline 14 & 0.24192 & 1.5946 & 1.5946 & 1.5946 \\
\hline 15 & 0.25882 & 1.5981 & 1.5981 & 1.5981 \\
\hline 16 & 0.27564 & 1.6020 & 1.6020 & 1.6020 \\
\hline 17 & 0.29237 & 1.6061 & 1.6061 & 1.6061 \\
\hline 18 & 0.30902 & 1.6105 & 1.6105 & 1.6105 \\
\hline 19 & 0.32557 & 1.6151 & 1.6151 & 1.6151 \\
\hline 20 & 0.34202 & 1.6200 & 1.6200 & 1.6200 \\
\hline 21 & 0.35837 & 1.6252 & 1.6252 & 1.6252 \\
\hline 22 & 0.37461 & 1.6307 & 1.6307 & 1.6307 \\
\hline 23 & 0.39073 & 1.6365 & 1.6365 & 1.6365 \\
\hline 24 & 0.40674 & 1.6426 & 1.6426 & 1.6426 \\
\hline 25 & 0.42262 & 1.6490 & 1.6490 & 1.6490 \\
\hline 26 & 0.43837 & 1.6557 & 1.6557 & 1.6557 \\
\hline 27 & 0.45399 & 1.6627 & 1.6627 & 1.6627 \\
\hline 28 & 0.46947 & 1.6701 & 1.6701 & 1.6701 \\
\hline 29 & 0.48481 & 1.6777 & 1.6777 & 1.6777 \\
\hline 30 & 0.50000 & 1.6858 & 1.6857 & 1.6858 \\
\hline 31 & 0.51504 & 1.6941 & 1.6941 & 1.6941 \\
\hline 32 & 0.52992 & 1.7028 & 1.7028 & 1.7028 \\
\hline 33 & 0.54464 & 1.7119 & 1.7119 & 1.7119 \\
\hline 34 & 0.55919 & 1.7214 & 1.7214 & 1.7214 \\
\hline 35 & 0.57358 & 1.7312 & 1.7312 & 1.7312 \\
\hline 36 & 0.58779 & 1.7415 & 1.7415 & 1.7415 \\
\hline 37 & 0.60182 & 1.7522 & 1.7522 & 1.7522 \\
\hline 38 & 0.61566 & 1.7633 & 1.7632 & 1.7633 \\
\hline 39 & 0.62932 & 1.7748 & 1.7748 & 1.7748 \\
\hline 40 & 0.64279 & 1.7868 & 1.7867 & 1.7868 \\
\hline 41 & 0.65606 & 1.7992 & 1.7992 & 1.7992 \\
\hline 42 & 0.66913 & 1.8122 & 1.8121 & 1.8122 \\
\hline 43 & 0.68200 & 1.8256 & 1.8256 & 1.8256 \\
\hline 44 & 0.69466 & 1.8396 & 1.8395 & 1.8396 \\
\hline 45 & 0.70711 & 1.8541 & 1.8540 & 1.8541 \\
\hline 46 & 0.71934 & 1.8691 & 1.8691 & 1.8691 \\
\hline 47 & 0.73135 & 1.8848 & 1.8847 & 1.8848 \\
\hline 48 & 0.74314 & 1.9011 & 1.9009 & 1.9011 \\
\hline 59 & 0.75471 & 1.9180 & 1.9178 & 1.9180 \\
\hline 50 & 0.76604 & 1.9356 & 1.9354 & 1.9356 \\
\hline 52 & 0.77715 & 1.9539 & 1.9536 & 1.9539 \\
\hline 53 & 0.78801 & 1.9729 & 1.9726 & 1.9729 \\
\hline & & & & \\
\hline 2964 & 1.9927 & 1.9923 & 1.9927 \\
\hline
\end{tabular}

Table 1. Values of the functions $\mathrm{K}$ (part two)

\begin{tabular}{|c|c|c|c|c|}
\hline$\theta\left(^{\circ}\right)$ & $k=\sin \theta$ & $\mathrm{K}(k)$ & $\mathrm{K}_{0}(k)$ & $\mathrm{K}_{1}(k)$ \\
\hline 54 & 0.80902 & 2.0133 & 2.0128 & 2.0133 \\
\hline 55 & 0.81915 & 2.0347 & 2.0341 & 2.0347 \\
\hline 56 & 0.82904 & 2.0571 & 2.0564 & 2.0571 \\
\hline 57 & 0.83867 & 2.0804 & 2.0795 & 2.0804 \\
\hline 58 & 0.84805 & 2.1047 & 2.1037 & 2.1047 \\
\hline 59 & 0.85717 & 2.1300 & 2.1288 & 2.1300 \\
\hline 60 & 0.86603 & 2.1565 & 2.1551 & 2.1565 \\
\hline 61 & 0.87462 & 2.1842 & 2.1825 & 2.1842 \\
\hline 62 & 0.88295 & 2.2132 & 2.2111 & 2.2132 \\
\hline 63 & 0.89101 & 2.2435 & 2.2410 & 2.2435 \\
\hline 64 & 0.89879 & 2.2754 & 2.2723 & 2.2754 \\
\hline 65 & 0.90631 & 2.3088 & 2.3051 & 2.3088 \\
\hline 66 & 0.91355 & 2.3439 & 2.3394 & 2.3439 \\
\hline 67 & 0.92050 & 2.3809 & 2.3754 & 2.3809 \\
\hline 68 & 0.92718 & 2.4198 & 2.4132 & 2.4198 \\
\hline 69 & 0.93358 & 2.4610 & 2.4530 & 2.4610 \\
\hline 70 & 0.93969 & 2.5046 & 2.4948 & 2.5045 \\
\hline 70.5 & 0.94264 & 2.5273 & 2.5165 & 2.5273 \\
\hline 71 & 0.94552 & 2.5507 & 2.5389 & 2.5507 \\
\hline 71.5 & 0.94832 & 2.5749 & & 2.5749 \\
\hline 72 & 0.95106 & 2.5998 & & 2.5998 \\
\hline 72.5 & 0.95372 & 2.6256 & & 2.6255 \\
\hline 73 & 0.95630 & 2.6521 & & 2.6521 \\
\hline 73.5 & 0.95882 & 2.6796 & & 2.6796 \\
\hline 74 & 0.96126 & 2.7081 & & 2.7081 \\
\hline 74.5 & 0.96363 & 2.7375 & & 2.7375 \\
\hline 75 & 0.96593 & 2.7681 & & 2.7680 \\
\hline 75.5 & 0.96815 & 2.7998 & & 2.7997 \\
\hline 76 & 0.97030 & 2.8327 & & 2.8326 \\
\hline 76.5 & 0.97237 & 2.8669 & & 2.8669 \\
\hline 77 & 0.97437 & 2.9026 & & 2.9025 \\
\hline 77.5 & 0.97630 & 2.9397 & & 2.9397 \\
\hline 78 & 0.97815 & 2.9786 & & 2.9785 \\
\hline 78.5 & 0.97992 & 3.0192 & & 3.0191 \\
\hline 79 & 0.98163 & 3.0617 & & 3.0616 \\
\hline 79.5 & 0.98325 & 3.1064 & & 3.1063 \\
\hline 80 & 0.98481 & 3.1534 & & 3.1533 \\
\hline 80.2 & 0.98541 & 3.1729 & & 3.1727 \\
\hline 80.4 & 0.98600 & 3.1928 & & 3.1927 \\
\hline 80.6 & 0.98657 & 3.2132 & & 3.2130 \\
\hline 80.8 & 0.98714 & 3.2340 & & 3.2338 \\
\hline 81 & 0.98769 & 3.2553 & & 3.2551 \\
\hline 81.2 & 0.98823 & 3.2771 & & 3.2769 \\
\hline 81.4 & 0.98876 & 3.2995 & & 3.2992 \\
\hline 81.6 & 0.98927 & 3.3223 & & 3.3221 \\
\hline 81.8 & 0.98978 & 3.3458 & & 3.3455 \\
\hline 82 & 0.99027 & 3.3699 & & 3.3696 \\
\hline 82.2 & 0.99075 & 3.3946 & & 3.3942 \\
\hline 82.4 & 0.99122 & 3.4199 & & 3.4196 \\
\hline 82.6 & 0.99167 & 3.4460 & & 3.4456 \\
\hline 82.8 & 0.99211 & 3.4728 & & 3.4724 \\
\hline 83 & 0.99255 & 3.5004 & & 3.4999 \\
\hline 83.2 & 0.99297 & 3.5288 & & 3.5283 \\
\hline 83.4 & 0.99337 & 3.5581 & & 3.5575 \\
\hline & & & & \\
\hline 5
\end{tabular}


Table 1. Values of the functions $\mathbf{K}$ (part three)

\begin{tabular}{|c|c|c|c|c|}
\hline$\theta\left(^{\circ}\right)$ & $k=\sin \theta$ & $\mathrm{K}(k)$ & $\mathrm{K}_{0}(k)$ & $\mathrm{K}_{1}(k)$ \\
\hline 83.6 & 0.99377 & 3.5884 & & 3.5877 \\
\hline 83.8 & 0.99415 & 3.6196 & & 3.6188 \\
\hline 84 & 0.99452 & 3.6519 & & 3.6510 \\
\hline 84.2 & 0.99488 & 3.6852 & & 3.6843 \\
\hline 84.4 & 0.99523 & 3.7198 & & 3.7187 \\
\hline 84.6 & 0.99556 & 3.7557 & & 3.7545 \\
\hline 84.8 & 0.99588 & 3.7930 & & 3.7916 \\
\hline 85 & 0.99619 & 3.8317 & & 3.8302 \\
\hline 85.2 & 0.99649 & 3.8721 & & 3.8704 \\
\hline 85.4 & 0.99678 & 3.9142 & & 3.9122 \\
\hline 85.6 & 0.99705 & 3.9583 & & 3.9560 \\
\hline 85.8 & 0.99731 & 4.0044 & & 4.0018 \\
\hline 86 & 0.99756 & 4.0528 & & 4.0498 \\
\hline 86.2 & 0.99780 & 4.1037 & & 4.1003 \\
\hline 86.4 & 0.99803 & 4.1574 & & 4.1535 \\
\hline 86.6 & 0.99824 & 4.2142 & & 4.2097 \\
\hline 86.8 & 0.99844 & 4.2744 & & 4.2692 \\
\hline 87 & 0.99863 & 4.3387 & & 4.3325 \\
\hline 87.2 & 0.99881 & 4.4073 & & 4.4001 \\
\hline 87.4 & 0.99897 & 4.4811 & & 4.4726 \\
\hline 87.6 & 0.99912 & 4.5609 & & 4.5507 \\
\hline 87.8 & 0.99926 & 4.6477 & & 4.6354 \\
\hline 88 & 0.99939 & 4.7427 & & 4.7277 \\
\hline 88.2 & 0.99951 & 4.8478 & & 4.8293 \\
\hline 88.4 & 0.99961 & 4.9654 & & \\
\hline 88.6 & 0.99970 & 5.0988 & & \\
\hline 88.8 & 0.99978 & 5.2527 & & \\
\hline 89 & 0.99985 & 5.4329 & & \\
\hline 89.1 & 0.99988 & 5.5402 & & \\
\hline 89.2 & 0.99990 & 5.6579 & & \\
\hline 89.3 & 0.99993 & 5.7914 & & \\
\hline 89.4 & 0.99995 & 5.9455 & & \\
\hline 89.5 & 0.99996 & 6.1278 & & \\
\hline 89.6 & 0.99998 & 6.3509 & & \\
\hline 89.7 & 0.99999 & 6.6385 & & \\
\hline 89.8 & 0.99999 & 7.0440 & & \\
\hline 89.9 & 1.00000 & 7.7371 & & \\
\hline 90 & 1.00000 & $\infty$ & & \\
\hline & & & & \\
\hline
\end{tabular}

The values strings contained in the last two columns of the previous table were canceled where each of the two closed analytic formulas proposed for the approximation of the Legendre complete elliptic integral of the first kind $\mathrm{K}(k)$ gives too great errors for being still accepted in the usual mathematical or technical calculus. The same procedure will be applied in the case of the following table, for the same reason, concerning the accuracy of the values given by each of the other two closed analytic formulas proposed for the approximation of the Legendre complete elliptic integral of the second kind $\mathrm{E}(k)$. The accuracy analysis of the two sets of formulas will be performed in the following chapter (no. 3). In the chapter 4 some series representations for the exact functions and for both sets of approximation, as well as for all their first order derivatives, will be given.
Table 2. Values of the functions $E$ (part one)

\begin{tabular}{|c|c|c|c|c|}
\hline$\overline{\theta\left(^{\circ}\right)}$ & $k=\sin \theta$ & $\overline{\mathrm{E}(k)}$ & $\overline{\mathrm{E}_{0}(k)}$ & $\overline{\mathrm{E}_{1}(k)}$ \\
\hline 0 & 0.00000 & 1.5708 & 1.5708 & 1.5708 \\
\hline 1 & 0.01745 & 1.5707 & 1.5707 & 1.5707 \\
\hline 2 & 0.03490 & 1.5703 & 1.5703 & 1.5703 \\
\hline 3 & 0.05234 & 1.5697 & 1.5697 & 1.5697 \\
\hline 4 & 0.06976 & 1.5689 & 1.5689 & 1.5689 \\
\hline 5 & 0.08716 & 1.5678 & 1.5678 & 1.5678 \\
\hline 6 & 0.10453 & 1.5665 & 1.5665 & 1.5665 \\
\hline 7 & 0.12187 & 1.5649 & 1.5649 & 1.5649 \\
\hline 8 & 0.13917 & 1.5632 & 1.5632 & 1.5632 \\
\hline 9 & 0.15643 & 1.5611 & 1.5611 & 1.5611 \\
\hline 10 & 0.17365 & 1.5589 & 1.5589 & 1.5589 \\
\hline 11 & 0.19081 & 1.5564 & 1.5564 & 1.5564 \\
\hline 12 & 0.20791 & 1.5537 & 1.5537 & 1.5537 \\
\hline 13 & 0.22495 & 1.5507 & 1.5507 & 1.5507 \\
\hline 14 & 0.24192 & 1.5476 & 1.5476 & 1.5476 \\
\hline 15 & 0.25882 & 1.5442 & 1.5442 & 1.5442 \\
\hline 16 & 0.27564 & 1.5405 & 1.5405 & 1.5405 \\
\hline 17 & 0.29237 & 1.5367 & 1.5367 & 1.5367 \\
\hline 18 & 0.30902 & 1.5326 & 1.5326 & 1.5326 \\
\hline 19 & 0.32557 & 1.5283 & 1.5283 & 1.5283 \\
\hline 20 & 0.34202 & 1.5238 & 1.5238 & 1.5238 \\
\hline 21 & 0.35837 & 1.5191 & 1.5191 & 1.5191 \\
\hline 22 & 0.37461 & 1.5141 & 1.5141 & 1.5141 \\
\hline 23 & 0.39073 & 1.5090 & 1.5090 & 1.5090 \\
\hline 24 & 0.40674 & 1.5037 & 1.5037 & 1.5037 \\
\hline 25 & 0.42262 & 1.4981 & 1.4981 & 1.4981 \\
\hline 26 & 0.43837 & 1.4924 & 1.4924 & 1.4924 \\
\hline 27 & 0.45399 & 1.4864 & 1.4864 & 1.4864 \\
\hline 28 & 0.46947 & 1.4803 & 1.4803 & 1.4803 \\
\hline 29 & 0.48481 & 1.4740 & 1.4740 & 1.4740 \\
\hline 30 & 0.50000 & 1.4675 & 1.4675 & 1.4675 \\
\hline 31 & 0.51504 & 1.4608 & 1.4608 & 1.4608 \\
\hline 32 & 0.52992 & 1.4539 & 1.4539 & 1.4539 \\
\hline 33 & 0.54464 & 1.4469 & 1.4469 & 1.4469 \\
\hline 34 & 0.55919 & 1.4397 & 1.4397 & 1.4397 \\
\hline 35 & 0.57358 & 1.4323 & 1.4323 & 1.4323 \\
\hline 36 & 0.58779 & 1.4248 & 1.4248 & 1.4248 \\
\hline 37 & 0.60182 & 1.4171 & 1.4171 & 1.4171 \\
\hline 38 & 0.61566 & 1.4092 & 1.4093 & 1.4092 \\
\hline 39 & 0.62932 & 1.4013 & 1.4013 & 1.4013 \\
\hline 40 & 0.64279 & 1.3931 & 1.3932 & 1.3931 \\
\hline 41 & 0.65606 & 1.3849 & 1.3849 & 1.3849 \\
\hline 42 & 0.66913 & 1.3765 & 1.3765 & 1.3765 \\
\hline 43 & 0.68200 & 1.3680 & 1.3680 & 1.3680 \\
\hline 44 & 0.69466 & 1.3594 & 1.3594 & 1.3594 \\
\hline 45 & 0.70711 & 1.3506 & 1.3507 & 1.3506 \\
\hline 46 & 0.71934 & 1.3418 & 1.3419 & 1.3418 \\
\hline 47 & 0.73135 & 1.3329 & 1.3330 & 1.3329 \\
\hline 48 & 0.74314 & 1.3238 & 1.3239 & 1.3238 \\
\hline 49 & 0.75471 & 1.3147 & 1.3148 & 1.3147 \\
\hline 50 & 0.76604 & 1.3055 & 1.3057 & 1.3055 \\
\hline 51 & 0.77715 & 1.2963 & 1.2964 & 1.2963 \\
\hline 52 & 0.78801 & 1.2870 & 1.2872 & 1.2870 \\
\hline 53 & 0.79864 & 1.2776 & 1.2778 & 1.2776 \\
\hline
\end{tabular}


Table 2. Values of the functions $E$ (part two)

\begin{tabular}{|c|c|c|c|c|}
\hline$\theta\left({ }^{\circ}\right)$ & $k=\sin \theta$ & $\mathrm{E}(k)$ & $\mathrm{E}_{0}(k)$ & $\mathrm{E}_{1}(k)$ \\
\hline 54 & 0.80902 & 1.2681 & 1.2684 & 1.2681 \\
\hline 55 & 0.81915 & 1.2587 & 1.2590 & 1.2587 \\
\hline 56 & 0.82904 & 1.2492 & 1.2496 & 1.2492 \\
\hline 57 & 0.83867 & 1.2397 & 1.2401 & 1.2397 \\
\hline 58 & 0.84805 & 1.2301 & 1.2307 & 1.2301 \\
\hline 59 & 0.85717 & 1.2206 & 1.2212 & 1.2206 \\
\hline 60 & 0.86603 & 1.2111 & 1.2118 & 1.2111 \\
\hline 61 & 0.87462 & 1.2015 & 1.2024 & 1.2015 \\
\hline 62 & 0.88295 & 1.1920 & 1.1930 & 1.1920 \\
\hline 63 & 0.89101 & 1.1826 & 1.1838 & 1.1826 \\
\hline 64 & 0.89879 & 1.1732 & 1.1745 & 1.1732 \\
\hline 65 & 0.90631 & 1.1638 & 1.1654 & 1.1638 \\
\hline 66 & 0.91355 & 1.1545 & 1.1564 & 1.1545 \\
\hline 67 & 0.92050 & 1.1453 & 1.1475 & 1.1453 \\
\hline 68 & 0.92718 & 1.1362 & 1.1387 & 1.1362 \\
\hline 69 & 0.93358 & 1.1272 & 1.1301 & 1.1273 \\
\hline 70 & 0.93969 & 1.1184 & 1.1217 & 1.1184 \\
\hline 70.5 & 0.94264 & 1.1140 & 1.1176 & 1.1140 \\
\hline 71 & 0.94552 & 1.1096 & 1.1135 & 1.1096 \\
\hline 71.5 & 0.94832 & 1.1053 & & 1.1053 \\
\hline 72 & 0.95106 & 1.1011 & & 1.1011 \\
\hline 72.5 & 0.95372 & 1.0968 & & 1.0968 \\
\hline 73 & 0.95630 & 1.0927 & & 1.0927 \\
\hline 73.5 & 0.95882 & 1.0885 & & 1.0885 \\
\hline 74 & 0.96126 & 1.0844 & & 1.0844 \\
\hline 74.5 & 0.96363 & 1.0804 & & 1.0804 \\
\hline 75 & 0.96593 & 1.0764 & & 1.0764 \\
\hline 75.5 & 0.96815 & 1.0725 & & 1.0725 \\
\hline 76 & 0.97030 & 1.0686 & & 1.0686 \\
\hline 76.5 & 0.97237 & 1.0648 & & 1.0648 \\
\hline 77 & 0.97437 & 1.0611 & & 1.0611 \\
\hline 77.5 & 0.97630 & 1.0574 & & 1.0574 \\
\hline 78 & 0.97815 & 1.0538 & & 1.0538 \\
\hline 78.5 & 0.97992 & 1.0502 & & 1.0503 \\
\hline 79 & 0.98163 & 1.0468 & & 1.0468 \\
\hline 79.5 & 0.98325 & 1.0434 & & 1.0435 \\
\hline 80 & 0.98481 & 1.0401 & & 1.0402 \\
\hline 80.2 & 0.98541 & 1.0388 & & 1.0389 \\
\hline 80.4 & 0.98600 & 1.0375 & & 1.0376 \\
\hline 80.6 & 0.98657 & 1.0363 & & 1.0364 \\
\hline 80.8 & 0.98714 & 1.0350 & & 1.0351 \\
\hline 81 & 0.98769 & 1.0338 & & 1.0339 \\
\hline 81.2 & 0.98823 & 1.0326 & & 1.0327 \\
\hline 81.4 & 0.98876 & 1.0314 & & 1.0315 \\
\hline 81.6 & 0.98927 & 1.0302 & & 1.0303 \\
\hline 81.8 & 0.98978 & 1.0290 & & 1.0292 \\
\hline 82 & 0.99027 & 1.0278 & & 1.0280 \\
\hline 82.2 & 0.99075 & 1.0267 & & 1.0269 \\
\hline 82.4 & 0.99122 & 1.0256 & & 1.0258 \\
\hline 82.6 & 0.99167 & 1.0245 & & 1.0247 \\
\hline 82.8 & 0.99211 & 1.0234 & & 1.0236 \\
\hline 83 & 0.99255 & 1.0223 & & 1.0226 \\
\hline 83.2 & 0.99297 & 1.0213 & & 1.0215 \\
\hline 83.4 & 0.99337 & 1.0202 & & 1.0205 \\
\hline & & & & \\
\hline
\end{tabular}

Table 2. Values of the functions $\mathrm{E}$ (part three)

\begin{tabular}{|c|c|c|c|c|}
\hline$\theta\left(^{\circ}\right)$ & $k=\sin \theta$ & $\mathrm{E}(k)$ & $\mathrm{E}_{0}(k)$ & $\mathrm{E}_{1}(k)$ \\
\hline 83.6 & 0.99377 & 1.0192 & & 1.0196 \\
\hline 83.8 & 0.99415 & 1.0182 & & 1.0186 \\
\hline 84 & 0.99452 & 1.0172 & & 1.0176 \\
\hline 84.2 & 0.99488 & 1.0163 & & 1.0167 \\
\hline 84.4 & 0.99523 & 1.0153 & & 1.0158 \\
\hline 84.6 & 0.99556 & 1.0144 & & 1.0150 \\
\hline 84.8 & 0.99588 & 1.0135 & & 1.0141 \\
\hline 85 & 0.99619 & 1.0127 & & 1.0133 \\
\hline 85.2 & 0.99649 & 1.0118 & & 1.0125 \\
\hline 85.4 & 0.99678 & 1.0110 & & 1.0118 \\
\hline 85.6 & 0.99705 & 1.0102 & & 1.0110 \\
\hline 85.8 & 0.99731 & 1.0094 & & 1.0103 \\
\hline 86 & 0.99756 & 1.0086 & & 1.0097 \\
\hline 86.2 & 0.99780 & 1.0079 & & 1.0091 \\
\hline 86.4 & 0.99803 & 1.0072 & & 1.0085 \\
\hline 86.6 & 0.99824 & 1.0065 & & 1.0080 \\
\hline 86.8 & 0.99844 & 1.0059 & & 1.0075 \\
\hline 87 & 0.99863 & 1.0053 & & 1.0071 \\
\hline 87.2 & 0.99881 & 1.0047 & & 1.0067 \\
\hline 87.4 & 0.99897 & 1.0041 & & 1.0064 \\
\hline 87.6 & 0.99912 & 1.0036 & & 1.0062 \\
\hline 87.8 & 0.99926 & 1.0031 & & 1.0060 \\
\hline 88 & 0.99939 & 1.0026 & & 1.0060 \\
\hline 88.2 & 0.99951 & 1.0021 & & 1.0061 \\
\hline 88.4 & 0.99961 & 1.0017 & & \\
\hline 88.6 & 0.99970 & 1.0014 & & \\
\hline 88.8 & 0.99978 & 1.0010 & & \\
\hline 89 & 0.99985 & 1.0008 & & \\
\hline 89.1 & 0.99988 & 1.0006 & & \\
\hline 89.2 & 0.99990 & 1.0005 & & \\
\hline 89.3 & 0.99993 & 1.0004 & & \\
\hline 89.4 & 0.99995 & 1.0003 & & \\
\hline 89.5 & 0.99996 & 1.0002 & & \\
\hline 89.6 & 0.99998 & 1.0001 & & \\
\hline 89.7 & 0.99999 & 1.0001 & & \\
\hline 89.8 & 0.99999 & 1.0000 & & \\
\hline 89.9 & 1.00000 & 1.0000 & & \\
\hline 90 & 1.00000 & 1.0000 & & \\
\hline & & & & \\
\hline
\end{tabular}

In the comparative tables 1 and 2 , the $4 D$ (four digit) exact values of both Legendre complete elliptic integrals reproduced from special functions tables [6], as well as their $4 D$ approximate values obtained by applying the two sets of proposed closed analytic formulas were given (all versus the respective elliptic integrals modulus, $k=\sin \theta$ ). It is to be noticed that both sets of approximate formulas are not given by spline or regression functions, but by asymptotic expansions, the respective expressions having a remarkable simplicity and accuracy. The identity with the exact functions is satisfied for the left end $k=0\left(\theta=0^{\circ}\right)$ of the domain. As one can see, the second set of functions $\left(\mathrm{K}_{1}, \mathrm{E}_{1}\right)$, although something more intricate, gives more accurate values than the first one $\left(\mathrm{K}_{0}, \mathrm{E}_{0}\right)$ and extends itself more closely to the right end $k=1\left(\theta=90^{\circ}\right)$ of the domain. 


\section{THE ACCURACY EVALUATION \\ OF THE TWO SETS OF FORMULAS}

Let define the following relative error functions: $\varepsilon_{\mathrm{K}_{0}}(k)=\mathrm{K}_{0}(k) / \mathrm{K}(k)-1 ; \quad \varepsilon_{\mathrm{K}_{1}}(k)=\mathrm{K}_{1}(k) / \mathrm{K}(k)-1$, for both sets of approximation of the first kind integral and $\varepsilon_{\mathrm{E}_{0}}(k)=\mathrm{E}_{0}(k) / \mathrm{E}(k)-1 ; \quad \varepsilon_{\mathrm{E}_{1}}(k)=\mathrm{E}_{1}(k) / \mathrm{E}(k)-1$, for both sets of approximation of the second kind integral. Their values are given in the table 3, being expressed in thousandths (\%o). These errors were calculated for the first set $\left(\mathrm{K}_{0}\right.$ and $\left.\mathrm{E}_{0}\right)$ only in the field $\theta \in\left[54^{\circ}, 71^{\circ}\right]$ of the domain, with an increment of $1^{\circ}$, while for the second set $\left(\mathrm{K}_{1}\right.$ and $\left.\mathrm{E}_{1}\right)$ only in the field $\theta \in\left[84^{\circ} .8,88^{\circ} .2\right]$, with an increment of $0^{\circ} .2$, like in the above tables 1 and 2 .

Table 3. Relative errors $\varepsilon$ distribution

\begin{tabular}{|c|c|c|c|c|c|}
\hline$\theta\left(^{\circ}\right)$ & $k=\sin \theta$ & $\varepsilon_{\mathrm{K}_{0}}(\%)$ & $\varepsilon_{\mathrm{K}_{1}}(\%)$ & $\varepsilon_{\mathrm{E}_{0}}(\%)$ & $\varepsilon_{\mathrm{E}_{1}}(\%)$ \\
\hline & & & & & \\
\hline 54 & 0.80902 & -0.250 & & +0.255 & \\
\hline 55 & 0.81915 & -0.272 & & +0.243 & \\
\hline 56 & 0.82904 & -0.353 & & +0.293 & \\
\hline 57 & 0.83867 & -0.420 & & +0.334 & \\
\hline 58 & 0.84805 & -0.497 & & +0.454 & \\
\hline 59 & 0.85717 & -0.558 & & +0.502 & \\
\hline 60 & 0.86603 & -0.669 & & +0.566 & \\
\hline 61 & 0.87462 & -0.799 & & +0.742 & \\
\hline 62 & 0.88295 & -0.961 & & +0.874 & \\
\hline 63 & 0.89101 & -1.118 & & +0.973 & \\
\hline 64 & 0.89879 & -1.366 & & +1.135 & \\
\hline 65 & 0.90631 & -1.619 & & +1.377 & \\
\hline 66 & 0.91355 & -1.918 & & +1.627 & \\
\hline 67 & 0.92050 & -2.299 & & +1.900 & \\
\hline 68 & 0.92718 & -2.709 & & +2.215 & \\
\hline 69 & 0.93358 & -3.253 & & +2.573 & \\
\hline 70 & 0.93969 & -3.907 & & +2.959 & \\
\hline 71 & 0.94552 & -4.642 & & +3.525 & \\
\hline & & - & & - & \\
\hline 84.8 & 0.99588 & - & -0.369 & - & +0.607 \\
\hline 85 & 0.99619 & - & -0.396 & - & +0.592 \\
\hline 85.2 & 0.99649 & - & -0.451 & - & +0.705 \\
\hline 85.4 & 0.99678 & - & -0.500 & - & +0.748 \\
\hline 85.6 & 0.99705 & - & -0.582 & - & +0.823 \\
\hline 85.8 & 0.99731 & - & -0.652 & - & +0.932 \\
\hline 86 & 0.99756 & - & -0.737 & - & +1.076 \\
\hline 86.2 & 0.99780 & - & -0.832 & - & +1.160 \\
\hline 86.4 & 0.99803 & - & -0.945 & - & +1.284 \\
\hline 86.6 & 0.99824 & - & -1.077 & - & +1.453 \\
\hline 86.8 & 0.99844 & - & -1.214 & - & +1.571 \\
\hline 87 & 0.99863 & - & -1.421 & - & +1.743 \\
\hline 87.2 & 0.99881 & - & -1.626 & - & +1.976 \\
\hline 87.4 & 0.99897 & - & -1.894 & - & +2.275 \\
\hline 87.6 & 0.99912 & - & -2.234 & - & +2.553 \\
\hline 87.8 & 0.99926 & - & -2.655 & - & +2.922 \\
\hline 88 & 0.99939 & - & -3.156 & - & +3.397 \\
\hline 88.2 & 0.99951 & - & -3.808 & - & +4.004 \\
\hline & & & & & \\
\hline & & & & & \\
\hline
\end{tabular}

\section{SOME COMPARATIVE SERIES REPRESENTATIONS}

Expanding into power series, one obtains for the complete elliptic integrals the following set of representations [5-7]:

$$
\begin{array}{r}
\mathrm{K}(k)=\frac{\pi}{2}\left(1+\frac{1}{4} k^{2}+\frac{9}{64} k^{4}+\frac{25}{256} k^{6}+\frac{1225}{16384} k^{8}+\frac{3969}{65536} k^{10}+\right. \\
\left.\quad+\frac{53361}{1048576} k^{12}+\frac{184041}{4194304} k^{14}+\frac{41409225}{1073741824} k^{16}+\ldots\right) ; \\
=\frac{\pi}{2}\left\{1+\sum_{n=1}^{\infty}\left[\frac{1 \cdot 3 \cdot \ldots(2 n-1)}{2 \cdot 4 \cdot \ldots \cdot 2 n}\right]^{2} k^{2 n}\right\}=\frac{\pi}{2}\left\{1+\sum_{n=1}^{\infty}\left[\frac{(2 n-1) ! !}{2^{n} n !}\right]^{2} k^{2 n}\right\} ; \\
\mathrm{E}(k)=\frac{\pi}{2}\left(1-\frac{1}{4} k^{2}-\frac{3}{64} k^{4}-\frac{5}{256} k^{6}-\frac{175}{16384} k^{8}-\frac{441}{65536} k^{10}-\right. \\
\left.\quad-\frac{4851}{1048576} k^{12}-\frac{14157}{4194304} k^{14}-\frac{2760615}{1073741824} k^{16}-\ldots\right) ; \\
=\frac{\pi}{2}\left\{1-\sum_{n=1}^{\infty}\left[\frac{1 \cdot 3 \cdot \ldots(2 n-1)}{2 \cdot 4 \cdot \ldots \cdot 2 n}\right]^{2} \frac{k^{2 n}}{2 n-1}\right\}=\frac{\pi}{2}\left\{1-\sum_{n=1}^{\infty}\left[\frac{(2 n-1) ! !}{2^{n} n !}\right]^{2} \frac{k^{2 n}}{2 n-1}\right\} .
\end{array}
$$

Proceeding in the same manner, we get for the first set of approximate functions (the most inaccurate) the expansions

$$
\begin{aligned}
& \mathrm{K}_{0}(k)=\frac{\pi}{2}\left(1+\frac{1}{4} k^{2}+\frac{9}{64} k^{4}+\frac{25}{256} k^{6}+\frac{1222}{16384} k^{8}+\ldots\right) ; \\
& \mathrm{E}_{0}(k)=\frac{\pi}{2}\left(1-\frac{1}{4} k^{2}-\frac{3}{64} k^{4}-\frac{5}{256} k^{6}-\frac{172}{16384} k^{8}-\ldots\right),
\end{aligned}
$$

for the $2^{\text {nd }}$ set being practically identical with the exact ones

$$
\begin{aligned}
\mathrm{K}_{1}(k) & =\frac{\pi}{2}\left(1+\frac{1}{4} k^{2}+\frac{9}{64} k^{4}+\frac{25}{256} k^{6}+\frac{1225}{16384} k^{8}+\frac{3969}{65536} k^{10}+\right. \\
& \left.+\frac{53361}{1048576} k^{12}+\frac{184041}{4194304} k^{14}+\frac{41409222}{1073741824} k^{16}+\ldots\right) ; \\
\mathrm{E}_{1}(k) & =\frac{\pi}{2}\left(1-\frac{1}{4} k^{2}-\frac{3}{64} k^{4}-\frac{5}{256} k^{6}-\frac{175}{16384} k^{8}-\frac{441}{65536} k^{10}-\right. \\
& \left.-\frac{4851}{1048576} k^{12}-\frac{14157}{4194304} k^{14}-\frac{2760606}{1073741824} k^{16}-\ldots\right) .
\end{aligned}
$$

The difference with respect to the expansions of the exact functions begins at the terms in $k^{8}$ for the first set of approximation, and at the terms in $k^{16}$ for the second one.

For the first order derivatives of the exact functions we get

$$
\begin{aligned}
& \frac{d \mathrm{~K}(k)}{d k}=\frac{\mathrm{E}(k)}{k\left(1-k^{2}\right)}-\frac{\mathrm{K}(k)}{k}=\frac{\pi}{4} k\left(1+\frac{9}{8} k^{2}+\frac{75}{64} k^{4}+\frac{1225}{1024} k^{6}+\right. \\
& \left.+\frac{19845}{16384} k^{8}+\frac{160083}{131072} k^{10}+\frac{1288287}{1048576} k^{12}+\frac{41409225}{33554432} k^{14}+\ldots\right) ; \\
& =\frac{\pi}{4} \sum_{n=1}^{\infty}\left[\frac{1 \cdot 3 \cdot \ldots(2 n-1)}{2 \cdot 4 \cdot \ldots \cdot 2 n}\right]^{2} n k^{2 n-1}=\frac{\pi}{4} \sum_{n=1}^{\infty}\left[\frac{(2 n-1) ! !}{2^{n-1} n !}\right]^{2} n k^{2 n-1} ; \\
& \frac{d \mathrm{E}(k)}{d k}=\frac{\mathrm{E}(k)-\mathrm{K}(k)}{k}=-\frac{\pi}{4} k\left(1+\frac{3}{8} k^{2}+\frac{15}{64} k^{4}+\frac{175}{1024} k^{6}+\right. \\
& \left.+\frac{2205}{16384} k^{8}+\frac{14553}{131072} k^{10}+\frac{99099}{1048576} k^{12}+\frac{2760615}{33554432} k^{14}+\ldots\right) ; \\
& =-\frac{\pi}{4} \sum_{n=1}^{\infty}\left[\frac{1 \cdot 3 \cdot \ldots(2 n-1)}{2 \cdot 4 \cdot \ldots \cdot 2 n}\right]^{2} \frac{n k^{2 n-1}}{2 n-1}=-\frac{\pi}{4} \sum_{n=1}^{\infty}\left[\frac{(2 n-1) ! !}{2^{n-1} n !}\right]^{2} \frac{n k^{2 n-1}}{2 n-1} .
\end{aligned}
$$


Applying the previous two exact relations and using the four definitions from chapter 2 one obtains the expansions

$$
\begin{aligned}
& {\left[\frac{d \mathrm{~K}(k)}{d k}\right]_{0}=\frac{\pi}{4} k\left(1+\frac{9}{8} k^{2}+\frac{75}{64} k^{4}+\frac{1225.75}{1024} k^{6}+\ldots\right) ;} \\
& {\left[\frac{d \mathrm{E}(k)}{d k}\right]_{0}=-\frac{\pi}{4} k\left(1+\frac{3}{8} k^{2}+\frac{15}{64} k^{4}+\frac{174.25}{1024} k^{6}+\ldots\right),}
\end{aligned}
$$

for the first set of approximate functions, and respectively

$$
\begin{aligned}
& {\left[\frac{d \mathrm{~K}(k)}{d k}\right]_{1}=\frac{\pi}{4} k\left(1+\frac{9}{8} k^{2}+\frac{75}{64} k^{4}+\frac{1225}{1024} k^{6}+\frac{19845}{16384} k^{8}+\right.} \\
& \left.+\frac{160083}{131072} k^{10}+\frac{1288287}{1048576} k^{12}+\frac{41409226.125}{33554432} k^{14}+\ldots\right) ; \\
& {\left[\frac{d \mathrm{E}(k)}{d k}\right]_{1}=-\frac{\pi}{4} k\left(1+\frac{3}{8} k^{2}+\frac{15}{64} k^{4}+\frac{175}{1024} k^{6}+\frac{2205}{16384} k^{8}+\right.} \\
& \left.+\frac{14553}{131072} k^{10}+\frac{99099}{1048576} k^{12}+\frac{2760614.25}{33554432} k^{14}+\ldots\right),
\end{aligned}
$$

for the second set of approximate functions.

The difference with respect to the expansions of the first order derivatives of the exact functions begins at the terms in $k^{7}$ for the first set of approximation, and at the terms in $k^{15}$ for the second one, being much smaller than that for the expansions of the respective sets of approximate functions.

\section{GRAPHIC COMPARISON}

The variation curves of both Legendre complete elliptic integrals, as well as that of the two sets of new proposed closed analytic functions are graphically represented in the comparative figures 1 and 2 , all versus the angle $\theta$, expressed in sexagesimal degrees and given by the relation $\theta=\sin ^{-1} k, k$ being the modulus of these elliptic integrals.

In both figures the exact functions - K $(k)$, E $(k)$ - were represented by solid (continuous) black lines, the first set of approximation $-\mathrm{K}_{0}(k), \mathrm{E}_{0}(k)$ - by dashed black lines and the second set of approximation $-\mathrm{K}_{1}(k), \mathrm{E}_{1}(k)$ - by solid red lines respectively.

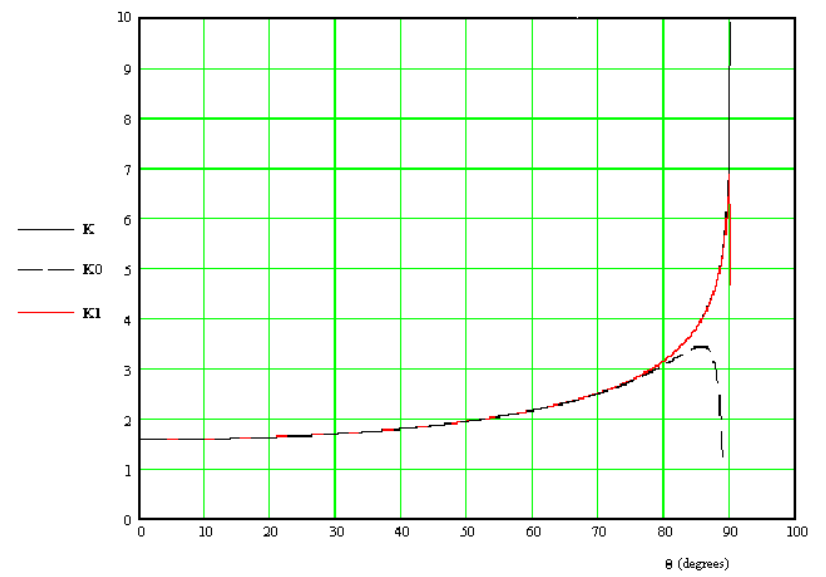

Figure 1. Comparison of the Legendre complete elliptic integral of the first kind $\mathrm{K}(\mathrm{k})$ with the new proposed closed analytic functions $K_{0}(k)$ and $K_{1}(k)$

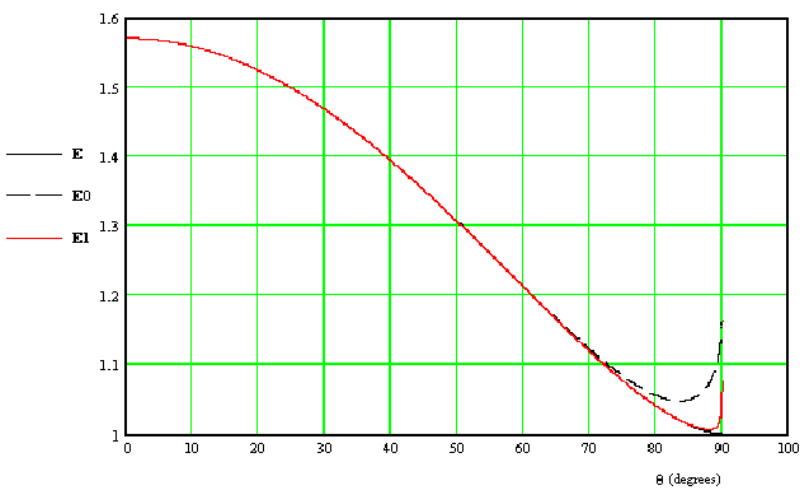

Figure 2. Comparison of the Legendre complete elliptic integral of the second kind $E(k)$ with the new proposed closed analytic functions $E_{0}(k)$ and $E_{1}(k)$

\section{CONCLUSION}

For reasons of accuracy it is recommended in the current mathematical and technical applications, to use the first set until $\theta=70^{\circ} .5(k=0.94264)$ only, and if it is necessary a better accuracy or a greater upper limit of the validity domain, to use the second set, but on no account beyond $\theta=88^{\circ} .2(k=0.99951)$.

\section{REFERENCES}

[1] Legendre, A. M., Tables of the complete and incomplete elliptic integrals. Reissued by K. Pearson, London, 1934.

[2] Heuman, C. A., Tables of complete elliptic integrals, J. Math. Physics, 20, pp. 127 - 206, 336, 1941.

[3] Hayashi, K., Tafeln der Besselschen, Theta-, Kugel- und anderen Funktionen, Berlin, 1930.

[4] Hayashi, K., Tafeln für die Differenzengleichung sowie für die Hyperbel-, Besselschen, elliptischen und anderen Funktionen, Berlin, 1933.

[5] Jahnke, E., Emde, F., Tables of Functions with Formulas and Curves, Dover Publications, New York, 1943.

[6] Jahnke, E., Emde, F., Lösch, F., Tafeln höherer Funktionen, sechste Auflage. Neubearbeitet von F. Lösch, B. G. Teubner Verlagsgesellschaft, Stuttgart, 1960.

[7] Gradshteyn, I. S., Ryzhik, I. M., Table of Integrals, Series, and Products. Fourth Edition Prepared by Yu. V. Geronimus/ M. Yu. Tseytlin, Academic Press, New York and London, 1965.

\section{Creative Commons Attribution License 4.0 (Attribution 4.0 International, CC BY 4.0)}

This article is published under the terms of the Creative Commons Attribution License 4.0

https://creativecommons.org/licenses/by/4.0/deed.en US 